\title{
Design and Control of Defense Robot Based On Virtual Reality
}

\author{
S. Navaneetha Krishna Kumar ${ }^{1}$, R. J. Praveen Kumar ${ }^{1}$, M. Raja Kumaran ${ }^{1}$, G. Valarmathy ${ }^{2}$ \\ ${ }^{1}$ Electronics and Communication Engineering, GKM College of Engineering and Technology \\ GKM Nagar, Perungalathur, Chennai-600 063, India \\ ${ }^{2}$ Assistant Professor, GKM College of Engineering and Technology \\ GKM Nagar, Perungalathur, Chennai-600 063, India
}

\begin{abstract}
This paper presents the tools that are essential for controlling a defense robot in a teleoperation system via internet using the virtual reality concept. In the process of controlling a defense robot based on remote access the most important criteria affecting the performance of robot is the delay time. This paper allows us to overcome the catastrophe of error that occurs during the operation of defense robot via internet. By utilizing the virtual reality concept the delay time across the defense robot can be minimized in an efficient way so that the defense robot performance can be improved to a certain extent. We implemented our work by conducting teleoperation experiments in different environment. The experimental result does go hand in hand with the above architecture.
\end{abstract}

Keywords: Defense robot, Delay time, Teleoperation, Virtual reality

\section{Introduction}

Based on current and emergence of new trends that could be evolved in near future the remote control technology is evolving periodically since the application involved in controlling various devices based on remote control technology that can be utilized for special purpose or general purpose the concept of virtual reality can be implemented [1].

The remote control (teleoperation) system of robot are widely used in many fields like industries, research, medical fields, education, entertainment and military purposes[5], In our work we are mainly focusing on implementing it in defense purpose. The teleoperation of robot is classified in to three categories: exploration rovers, unmanned ground vehicles and dangerous duties [6].

The exploration rover is a vehicle used to explore outer space and it is designed to move across the surface of a planet [7]. Rovers are created to land on another planet in the solar system, besides earth to find out information about the planet and to take samples. They can collect samples such as dust, rocks and even take pictures of the planet. An unmanned ground vehicle (UGV) is simply a vehicle that operates when it has a contact over the ground [8]. It could operate without the presence of human operator inside the vehicle. A dangerous duty robot is specifically used in the defense purpose where it is almost impossible for human to enter or operate $[9,10,11]$.

The most widely used technique for teleoperation purpose is the direct interface method [2]. The term direct interface basically defines the concept of controlling a device in this case a robot using a hand controller. But in this concept without using the implementation of direct interface another concept known as virtual reality is utilized. The term virtual reality is basically defined as creating an environment so that the user can feel that he is actually present there. Here the virtual reality technique is used because it involves lower operating cost than the other real situation $[12,13]$. Not only that in teleoperation of robot using other concepts delay time will be considerably high and by using virtual reality technique the delay time can be minimized to a certain extent $[14,15]$.

A new field of robotics known as telerobotics has fascinated the interest of various researchers in recent occasion [16]. This term refers to remotely controlling a robot through the internet. The advantage of using the above process is that it provides access virtually to resources present anywhere across the world. However by using internet [21] for controlling a robot the drawback is that, it does not provide the necessary quality of service and so there will be a loss in delivery of packets [4] and therefore it affects the performance of teleoperation system.

The serial communication between the transmitter and receiver in robot control is performed by Zigbee [3]. Zigbee is related to IEEE 802.15.4 which is a standard based protocol that provides the essential infrastructure required for the network. Here Zigbee provides the network and application layer whereas the 802.15 .4 provides physical layer and Mac layer. The applications of Zigbee are enormous across communication it can be used to communicate with a large number of devices and also there are enormous applications ideal for the self-configuring and self- healing capabilities of Zigbee networks.

- Energy Management and efficiency-For improving the information that are provided and to control the usage of energy so that the customers can have better service that helps to utilize the resources effectively.

- Building Automation- To provide more supportive security and home entertainment system from anywhere inside the block.

Zigbee has a interoperable nature so that these application can work together, this comes in handy because the device works more efficiently with greater benefits.

The advantages of using zigbee are it is highly secure, cost is considerably low and also it is a reliable and self-healing network which operates at low power. 


\section{Architecture for Designing Software}

The architecture for designing the software is based on the modules and communication between them using the request protocol, to ensure the safety of the user the controlling of robot is done remotely using networking system hence the connection between the server and clients are done using the transmitted data frames through instant network communications protocols.

\subsection{Establishment of Connection in the Server and Clients}

In the server station to control the robot by downloading the graphical interfaces and to transmit it in order the TCP/IP module is applied and to connect the defense robot with the server the wireless networking protocol is used. The server mainly focuses on power backup applications where major requirements are obtaining minimum data rate, and long battery life at minimum cost.

\subsection{Teleoperation System Architecture}

The architecture of Teleoperation in this system consists of the client PC Which is in remote place and the server defense robot that is communicating using the TCP/IP protocol module in this while the operator operating the defense robot using virtual reality concept the robot movements can be seen in client PC by using the camera attached in the robot, the images captured in the cameras are transmitted using internet.

\subsection{Concept of Remote Control and Virtual Reality}

In the teleoperation of the robot control the major problem is the delay in time across the system and also bandwidth therefore reducing the signal quality of the video hence the virtual reality technology is used to overcome these problems, the robot implemented in this case is defense robot, the advantages of the virtual reality interface are its user friendly nature, visual aids, customized display, impressive data presentation and the redundancy of data in the screen [17].

In the teleoperation of the robot control the quality of the video will be very low hence in this case the virtual reality technology will be helpful for the user to obtain the better view of this video with increase in the quality and also control the defense robot remotely. This technology also decreases the time delay that is presented in the networks.

\subsection{Forward Kinematics of Defense Robot}

In our concept the model of the defense robot utilized is a unicycle type. In this process configuration of defense robot is defined completely by three parameters $\mathrm{P}, \mathrm{Q}$ and $\theta$. The distance between two wheels is assumed as $\mathrm{D}$. The angular velocity of a defense robot around midpoint of wheel (MP) is assumed as $\omega$. The velocities of right and left wheel are assumed as $\mathrm{v}_{1}$ and $\mathrm{v}_{2}$ respectively and its respective angular velocities are assumed to be $\omega_{1}$ and $\omega_{2}$ respectively. The radius of the wheel is stated as r. Figure 1 shows the basic parameters of the unicycle type defense robot. The linear velocity $\mathrm{v}$ is given by the relation

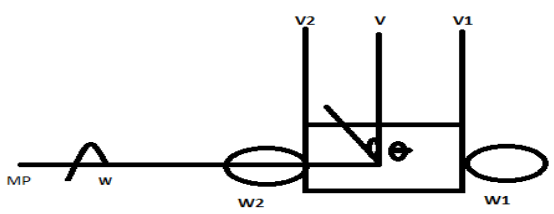

Figure 1: Kinematics of Defense Robot

$$
\begin{gathered}
\mathrm{v}=\frac{\mathrm{v} 1+\mathrm{v} 2}{2} \\
\mathrm{v}=\frac{\mathrm{r} \omega 1+\mathrm{r} \omega 2}{2}=\frac{\mathrm{r}(\omega 1+\omega 2)}{2}
\end{gathered}
$$

The angular velocity of the defense robot is given by:

$$
\widehat{\theta}=\frac{\mathrm{v} 1+\mathrm{v} 2}{\mathrm{D}}
$$

Where $v_{1}-v_{2}=r\left(\omega_{1}-\omega_{2}\right)$

$$
\theta=\frac{r(\omega 1+\omega 2)}{2}=\omega
$$

The kinematic model is given by the relations

$$
\begin{gathered}
\widehat{\mathrm{P}}=\mathrm{v} \cos \theta \\
\widehat{\mathrm{P}}=\mathrm{v} \sin \theta \\
\widehat{\theta}=\omega
\end{gathered}
$$

It is assumed that the robot wheel does not roll and slip, the direction of robot can never be sideways with respect to the wheels, that is:

$$
-\widehat{P} \sin \theta+\widehat{Q} \cos \theta=0
$$

\subsubsection{Path followed by the robot}

For path followed, an algorithm is proposed for tracking the target position $\mathrm{x}^{*}(\mathrm{t}) \cdot \mathrm{y}^{*}(\mathrm{t})$, the robot will keep up a certain distance $d^{*}$, this error between the position of robot and target position is found by the relation:

$$
\mathrm{e}=\sqrt{\left(\mathrm{P}^{*}-\mathrm{P}\right)^{2}+\left(\mathrm{Q}^{*}-\mathrm{Q}\right)^{2}}-\mathrm{d}^{2}
$$

The angle $\theta^{*}$ can be stated as given below:

$\theta^{*}=\tan ^{-1}\left(\frac{\mathrm{Q} *-\mathrm{Q}}{\mathrm{P} *-\mathrm{P}}\right)$

Generally, the kinematics model of a unicycle robot is utilized to rotate the routing wheel. The inputs of the robot are $\omega$ and $\mathrm{v}$ and the present rotating angle is the respective output and is given by $\theta$.

\subsection{Inverse Kinematics of Defense Robot}

The center of mass position in the wheels of the robot with respect to the turn angles are identified by the direct kinematic models, the expression of the direct kinematic models is expressed as: 


\section{International Journal of Science and Research (IJSR) \\ ISSN (Online): 2319-7064}

Index Copernicus Value (2013): 6.14 | Impact Factor (2014): 5.611

$$
\begin{gathered}
\widehat{P}=-\frac{r \sin \theta}{2} \widehat{\omega}_{1}-\frac{r \sin \theta}{2} \widehat{\omega}_{2} \\
\widehat{Q}=\frac{r \sin \theta}{2} \widehat{\omega}_{1}+\frac{r \sin \theta}{2} \widehat{\omega}_{2} \\
\widehat{\theta}=\frac{r}{D} \widehat{\omega}_{1}-\frac{r}{2} \widehat{\omega}_{2}
\end{gathered}
$$

To find the angular velocity of each wheel the inverse kinematics is used, the expression for the inverse kinematic model is expressed as:

$$
\begin{aligned}
& \widehat{\omega}_{1}=-\frac{2 v-D \omega}{2 r} \\
& \widehat{\omega}_{2}=-\frac{2 v-D \omega}{2 r}
\end{aligned}
$$

\section{Control Side}
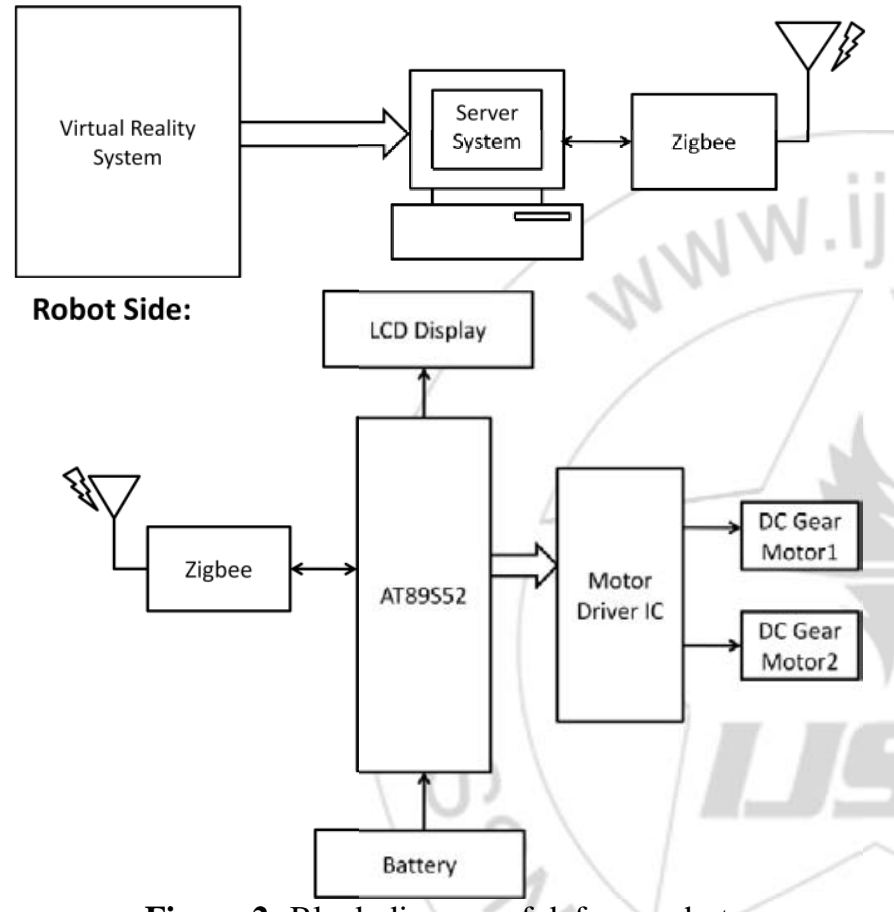

Figure 2: Block diagram of defense robot

\section{Hardware Design}

The basic block diagram to implement our proposed concept is give in figure 1.In the process of controlling the defense robot using remote access technique, the station of such access control consist of two standard parts.

\section{i. Application Server}

The Server which develops the information about an application from the server station and forwarded to the robot at the other end is called as Application Server. As liaison it reflects the information which is received from the client and converts to understandable instructions for the robot.

\section{ii. Application Client}

The client used by the hand operator and its application used for controlling and tele-operating the functions of robots. Here, for operating the functions some data information has been flowed.

\subsection{Configuration of Server PC}

Processor: Intel core i3 $(2.30 \mathrm{GHz})$

RAM: 3GB RAM

Ethernet Network Device: RTL8101E family PCI-E Fast Ethernet NIC

Wireless Network Device: Realtek RTL8187B Wireless 802.11g 54Mbps.

\subsection{Robot Activity Control}

In this concept mobile robot is designed using basic two wheeled land rover robot. Two wheels are proposed in order to move in different angles and dimensions. Normally, the robot can move in four different angles, hence the two wheels will tend to decide the direction based on turning of wheels. While controlling the motion of the robot it tends to communicate with the personal computer that is server.

After it is communicated with the server pc, it gets transmitted via the ZigBee module which here plays mainly as a transmitter. ZigBee module is the set based wireless sensor network.

Our main consideration in this paper is to avoid the delay time of packets given to the robot, hence we are using the virtual reality concept and interactions with the server pc and robotic system to get efficient information without data loss $[18,19]$.

\subsection{Description of Two Wheeled Mobile Robot}

The two wheeled mobile robot as described, it consists of two wheels and those wheels are controlled using the Dc gear motor for the purpose of controlling the motion of robot. Here the two wheels tend for the intention to drive over the direction controlled by the motor and also dummy wheels are provided for support at the rear as an insane. The logic of zeros and ones plays a controlling role in movement and direction of the two wheels that moves with the help of gear motor.

\subsubsection{Proportions of Two Wheeled Robot}

Radius of one wheel- $2.5 \mathrm{~cm}$

Distance between two wheels- $15 \mathrm{~cm}$

Weight- 100g

\subsubsection{Description about Structure of Robot}

Robot can be explained in co-ordinates structure, A and B are global coordinates. $\mathrm{A}_{1^{-}} \mathrm{B}_{1}$ are local coordinates. Our mobile robot is consisting of square shaped symmetrical body with two spherical wheels and centre of the body is "O". From the diagram the displacement of the body is $\mathrm{L}$ and radius is $\mathrm{R}$. here the angle that originate as $\theta$.

\subsection{Action of Controller}

Basically we get to use Atmel AT89S52 (Microcontroller), which is now widely reached and used most. Such microcontroller will get the information from the server $\mathrm{pc}$ via ZigBee module. From the received signal i.e. in the form 


\section{International Journal of Science and Research (IJSR) \\ ISSN (Online): 2319-7064 \\ Index Copernicus Value (2013): 6.14 | Impact Factor (2014): 5.611}

of coded information, controller will encode and generate the PWM signals to control the function of the wheels in the robot.

The functions of the wheels are normally controlled using logic of zeroes and ones encoded using the program or algorithm written via Server pc. Hence the two wheels can be controlled for the movement of robot in different directions, such as "UP", "DOWN", "LEFT", and "RIGHT".

Here, for the movement in different directions we are using more switches to control the movement. Also we were using our Mobile Robot for the defense purpose so; we are in need to enable another switch for defense purpose like "SHOOT".

Hence with the help of switches, we can switch over the direction of Robot. For example, if we want to move the robot in forward direction, the logic is 1010 it represents that $M 1=1, M 2=0, M 3=1, M 4=0$; hence due to M1 and M3 are positive, the two wheels will tends to move forward in direction. In case 2, when the logic is 0101.It represents that $\mathrm{M} 1=0, \mathrm{M} 2=1, \mathrm{M} 3=0, \mathrm{M} 4=1$; Hence due to $\mathrm{M} 2$ and $\mathrm{M} 4$ are positive and other two motors terminals are negative, therefore the two wheels will tend to move in backward direction .In case 3 , the logic is 1000 it represents that $M 1=1$, M2 $=0$, M3 $=0$, M4 $=0$; hence one of the wheel (right) remains idle so other wheel(left) tends to move forward because of its terminal is positive, hence it gets right in turn of movement. In case 4 the logic is 0010 . It represents that M1 $=0, M 2=0$, M3=1, M4=0; hence due to M3 is in positive terminal the right wheel only tends to move forward but left wheel remains quite. The above illustrated commands can be accurately understood by the table 1 mentioned below:

Table 1: Commands to determine direction of robot

\begin{tabular}{|c|c|c|c|c|}
\hline Condition & M1 & M2 & M3 & M4 \\
\hline Up & 1 & 0 & 1 & 0 \\
\hline Down & 0 & 1 & 0 & 1 \\
\hline Right & 1 & 0 & 0 & 0 \\
\hline Left & 0 & 0 & 1 & 0 \\
\hline Stop & 0 & 0 & 0 & 0 \\
\hline
\end{tabular}

\section{Interfacing of Virtual Reality and Tele- Robotic System}

In order to interface the virtual reality concept with the robotic concept, the virtual light is engendered via projector and focused on the wall. Such light projects the remote control access for robot movement. Using the digital image processing technique we can able to process the image projected on the wall and it gets compared with the predefined images which is already stored in the buffer.

Hence from the comparison of images, the instructions given in the image is passed into the controller and it tends the movement of motor in different directions. Normally virtual reality concept is used to avoid the delay time occurred in transmission of information for the control of robot, here the information is transferred via the light. The light transmission like lifi technology is mostly used for fast transmission and hence the data loss is minimized and transmitted efficiently so, the performances of robot get increased.
The operator here plays a role of controlling the commands to operate the robot. the main three task normally operator will do are;
i. Operator instructions
ii. Processing the image
iii. Simulating the robot

\subsection{Communication in Tele-Robotic Application}

The Tele-robotic application uses the internet communication to understand the data transmitted via Zigbee. Some factors are limited due to internet communication are bandwidth, efficiency, dropping of data, unwanted latency and delay in time because of long distance transmission.

To get rid of these problems, the virtual reality concept is coiled for fast transmission, so most of the problems get overcame. By using the man-robot interface inside our architecture we can overcome most part of problems aroused. Some protocols like TCP/IP can be used with the server and client to implement the long distance transmission [20].

\subsection{Description of Virtual Reality}

Various theories behind virtual reality led the human desire to go beyond the boundaries of existing world by accepting cyberspace. On achieving that we can interact with the virtual environment by all means in a simpler manner that could help to discover new forms of human machine interaction.

The objective is to go beyond the basic interaction with components such as keyboard and mouse that are utilized on a regular basis. This does not seem as a user-friendly way of working which pressures the people to get used to the demands of technology rather than the other way.

To obtain new form of the Human-Machine Interaction (HMI) Lumisense Virtual Reality board is utilized. In this the designer has the advantage to effortlessly, rapidly and flawlessly add with the microcontroller by UART to their applications. Figure 2 shows the basic virtual reality system implemented in our project.

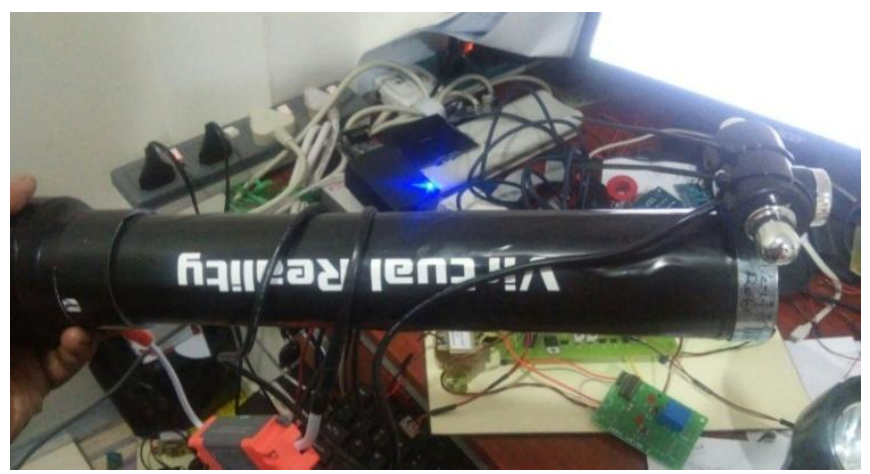




\section{International Journal of Science and Research (IJSR) \\ ISSN (Online): 2319-7064}

Index Copernicus Value (2013): 6.14 | Impact Factor (2014): 5.611

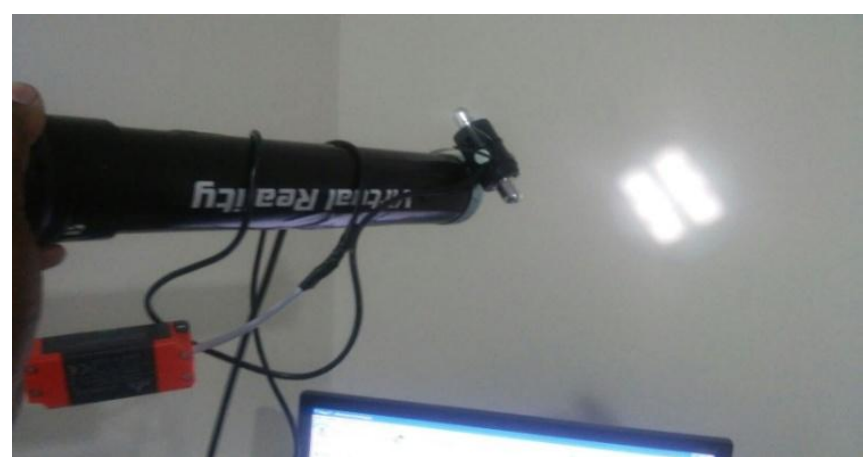

Figure 3: Virtual Reality Systems

\subsubsection{Specification}

The input specification of the virtual reality system are it requires a power supply of DC+12v 1 Amps and the USB for the Camera driver installation, whereas the output is a UART RS232 with a baud rate of 9600 . The output character for each condition touch is given in the table below.

With respect to the imaged touched a single character is sent to the UART which will be easier to connect or can be directly connected across any UART wireless transmitter.

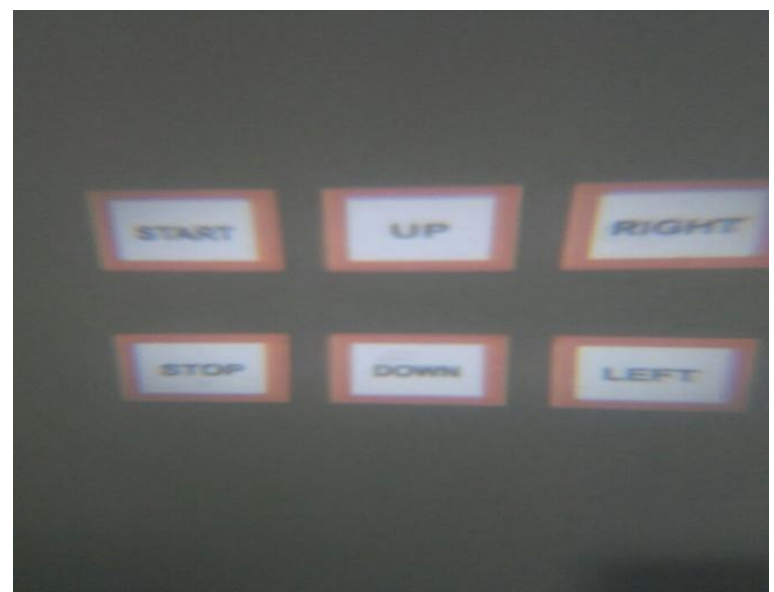

Figure 4: Condition touch of virtual reality system

\subsection{Implementation of our Robot}

On my reference to our project, we had studied some paper of different authors, where some gave about brief discussion of virtual reality based mobile robot it uses the VR concept for simple mobile robot. In another paper we referred about the collision free navigation for path planning. Some authors were involved only in simulating the output and not in real time implementation.

A few authors were used augmented reality concept of control. Hence following the above ideas we were implemented the virtual reality concept mobile robot for defense purpose. Such robot is used in military applications.

\section{Conclusion}

In this paper, we have presented a virtual reality system through which a robot can be controlled. The virtual reality concept is utilized in order to increase the delay in time across the robot to a certain extent. Also the wireless robot control is serially communicated by using the Zigbee module, the reason to use Zigbee module is for efficiency and accuracy and also provides stability, while using the Zigbee module the experimental results shows increased throughput and also the delay in time across the system is reduced considerably. It also allows us to control the robot in a 3D manner. It consists of three process first is the implementation of virtual reality system and the second course is serial communication using Zigbee and the third process is the operation of robot.

In the near future, we plan on implementing the concept by replacing virtual reality with augmented reality that could provide a major boost in operations such as remote manipulation and remote manufacturing, entertainment, education etc.

\section{References}

[1] Ibari Benaoumeur, Ahmed-foitih Zoubir, and Hanifi Elhachimi Amar Reda, "Remote control of mobile robot using the virtual reality, "International Journal of Electrical and Computer Engineering (IJECE), vol.5, No.5, October 2015, pp.1062 1074.

[2] Masmoudi. Mostefa, L. Kaddour El Boudadi, A. Loukil Khelf. Mohamed, Dahane. Amine, "Design of mobile robot teleoperation system based on virtual reality, " 2015, $3^{\text {rd }}$ International Conference on Control, Engineering and Information technology (CEIT).

[3] Mofeed Turkey Rashid, Abduladhem abdulkareem Ali, Ramzy Salim Ali, Luigi Fortuna, Mattia Frasca, Maria Gabriella Xibilia, "Wireless underwater mobile robot system based on zigbee, " 2012 International Conference on Future Communication Networks (ICFCN).

[4] Meha Sharma, Rewa Sharma, Kamna Ahuja, Swati Jha, "Design of an Intelligent Security Robot for Collision free Navigation Applications, " 2014 International Conference on Reality, Optimization and Information Technology-ICROIT, India, Feb 6-8 2014.

[5] Sheridan. Thomas B. "Telerobotics, automation, and Human Supervisory Control," 1992, MIT Press Cambridge, USA, Reading, MA.

[6] T.Fong, C.Thorpe, "Vehicle Teleoperation Interfaces", 2001, Autonomous robots, vol.11, no.1, pp.9-18, 2001.

[7] D.Carrier, W.David, "Soviet rover systems", in proceedings of the AIAA Space Programs and Technologies Conference, Huntsville, AL, USA, 1992.

[8] D.Gage, "UGV histort 101: A brief history of Unmanned Ground Vehicle (UGV) development efforts", 1995, Unmanned System Magazine, vol.13, no.3, pp.9-16, 1995.

[9] W.Whittaker and L.Champeny, "Conception and development of two mobile teleoperated system for TMI-2", in proceedings of the International Meeting and Topical Meeting TMI-2 Accident, American Nuclear Society, 1988.

[10]D.Hainsworth, “ Mine emergency Survey vehicle: Numbat", in Proceedings of the $25^{\text {th }}$ International Conference of Safety in Mines Research Institutes, Sep 1993, Pretoria, South Africa, pp, 7-18.

[11]T.Blackmon, L.Nguyen, C.Neveu, D.Rasmuseen., et al., "Virtual reality mapping system for Chernobyl accident 


\section{International Journal of Science and Research (IJSR) \\ ISSN (Online): 2319-7064}

Index Copernicus Value (2013): 6.14 | Impact Factor (2014): 5.611

site assessment", in proceedings of SPIE Conference on Human Vision and Electronic Imaging IV, San Jose, CA, pp. 338-345, 1999.

[12] D.Hainsworth, "Teleopertion user interfaces for mining robotics", 2001, Autonomous Robots, vol. 11, no 1, pp. 19-28.

[13] S.Jayarama, HI. Connache and KW. Lyons, 1997, "Virtual reality techniques", Computer-Aided Design, vol.29, no.8, pp.575-584.

[14] KP. Beier, "Web-Based virtual reality in design and manufacturing applications", in proceedings of First International Conference on Computer Applications and IT in the Maritime Industries, Germany, pp 45-55, 2001.

[15] D.Marini, R.Folgeriri, D.Gadia, and A.Rizzi, "Virtual Reality as a communication Process", 2012, Virtual Reality, vol.16, no.3, pp.233-241.

[16] JY.Lee, H.Kim and K.Kim, "A Web-enabled approach to featured-based modeling in a distributed and collaborative design environment", 2001, Concurrent Engineering, vol.9, no.1, pp. 74-87.

[17] Feng Cui, Ming-Lu Zhang, Bing-Qing Liu, "Decision of the, Emding Robot Based on Virtual Reality and Intelligent Decision System", 13-16 August 2006, Proceedings of the Fifth International Conference on Machine Learning and Cybernetics, Dailian, 1-42440060 0/06/\$20.00@2006.

[18] Tiam Hee Tee, Kok Seng Eu, Kian Meng Yap, Alan Marshall, Tsung-Han Lee, $20134^{\text {th }}$ International Conference on Intelligent Systems, Modelling and Simulation “ 3D Smart User Interactive System with Real-Time Responding Tele-Robotic Proprioceptive Information”,, 2166-0662/13 \$26.00 (C) 2013 IEEE DOI 10.1109/ISM.2013.90.

[19] Bambang R. "Development of Internet-telerobotics systems”, France, 2002, Proceedings of Information Systems Management Research Concentration.

[20] R.C.Luo, K.L.Su, "Networked intelligent robots through the internet: issues and opportunities", IEEE Proc.91 (3) (2003)371-382.

[21] R.Siegwart, K.Goldberg, "Robots on the web", IEEE Rob. Au-tom. Mag 7(March(1)) (2000)4 